\title{
BMJ Open Implementation and evaluation of a physical activity counselling programme in primary care among cancer survivors: SoDA study protocol
}

\author{
Famke Huizinga (D) , ${ }^{1}$ Nico-Derk Lodewijk Westerink, ${ }^{1}$ Annette J Berendsen, ${ }^{1}$ \\ Annemiek M E Walenkamp, ${ }^{2}$ Mathieu H G de Greef, ${ }^{3}$ Geertruida H de Bock, ${ }^{4}$ \\ Marjolein $Y$ Berger, ${ }^{1}$ Daan Brandenbarg ${ }^{1}$
}

To cite: Huizinga F, Westerink $\mathrm{N}$ $\mathrm{DL}$, Berendsen AJ, et al. Implementation and evaluation of a physical activity counselling programme in primary care among cancer survivors: SoDA study protocol. BMJ Open 2022;12:e060098. doi:10.1136/ bmjopen-2021-060098

- Prepublication history for this paper is available online. To view these files, please visit the journal online (http://dx.doi org/10.1136/bmjopen-2021 060098).

Received 13 December 2021 Accepted 09 February 2022

Check for updates

(c) Author(s) (or their employer(s)) 2022. Re-use permitted under CC BY-NC. No commercial re-use. See rights and permissions. Published by BMJ.

${ }^{1}$ General Practice and Elderly Care Medicine, University Medical Centre Groningen, Groningen, The Netherlands ${ }^{2}$ Medical Oncology, University Medical Centre Groningen, Groningen, The Netherlands ${ }^{3}$ Human Movement Sciences, University Medical Centre Groningen, Groningen, The Netherlands

${ }^{4}$ Epidemiology, University Medical Centre Groningen, Groningen, The Netherlands

Correspondence to Ms Famke Huizinga; f.huizinga@umcg.nl

\section{ABSTRACT}

Introduction Physical activity (PA) favourably affects various health outcomes in cancer survivors, but little is known about how to implement a PA programme in primary care. We therefore aim to implement and evaluate such a programme for cancer survivors in general practice. Methods and analyses The Stimulation of Daily Activity study is an implementation study with a single-arm longitudinal design in 15 Dutch general practices. Patients aged $\geq 18$ years who finished cancer treatment more than 6 months ago will be eligible for inclusion. The intervention will comprise six coaching sessions with the practice nurse in 9 months, seeking to increase PA in daily activities and using an activity tracker for goal setting and feedback. The Reach, Effectiveness, Adoption, Implementation and Maintenance framework will be used to evaluate implementation in terms of the health outcomes, extent of implementation and barriers and facilitators to implementation, using a mixed methods approach. Descriptive analyses and linear mixed model analyses will be performed on the quantitative data, while qualitative data from focus groups and interviews will be analysed by thematic analyses.

Ethics and dissemination The Medical Research Ethics Committee of the University Medical Centre Groningen, the Netherlands, concluded that this study was not subject to the Dutch Medical Research Involving Human Subjects Act (registration number: 201900586). The study results will be made available to patients and general practitioners via (inter)national publications and conferences, newsletters, public summaries and via (social) media.

\section{BACKGROUND}

Cancer survivors frequently suffer from persistent physical and psychosocial symptoms that are accompanied by perceived barriers to physical activity (PA) interventions, as illustrated in box 1. Given that PA interventions can favourably affect fatigue, depression, anxiety and physical function, ${ }^{1-8}$ it is unfortunate that most cancer survivors $(80 \%)$ do not achieve the levels recommended by the American College of Sports and Medicine. ${ }^{9}$
Strengths and limitations of this study

- This implementation research design allows us to constantly adapt the implementation process to what works best for the users involved (patients, general practitioners and practice nurses).

- Both quantitative and qualitative methods will be used for the evaluation at individual and organisational level.

- A limitation is the uncontrolled design of the study, preventing estimation of intervention effects.

To address this, PA interventions can be offered that support and motivate sedentary patients with unhealthy lifestyles; however, research shows that this group may not be reached. ${ }^{1011}$ Cancer survivors who take part in, and adhere to, PA programmes are more likely to have a higher education level, be nonsmokers, be more physically active in the past, consume less alcohol, have less psychological distress and receive more family support than non-participants. ${ }^{10} 11$ This suggests that PA interventions are predominantly appealing to people who are motivated to exercise and who already have a healthier lifestyle.

To target cancer survivors who are sedentary and have unhealthy lifestyles, interventions must overcome barriers to PA. Factors that enhance participation are programme availability and accessibility, social support, knowledge of PA guidelines, and counselling or advice. ${ }^{11} 12$ Individualised PA programmes that seek to increase daily activities at home with feedback and coaching may offer a solution for sedentary patients who are not intrinsically motivated to engage in extensive training. ${ }^{11} 13$ Primary care suits the implementation of such a programme not only because general practitioners (GPs) are located closer to where the patient lives but also because 


\section{Box 1 Illustrative case}

A 53-year-old man with a history of colorectal cancer 3 years ago. Since curative treatment with surgery and chemotherapy he feels tired and regularly worries about his health and future. He often stays home after work or at the weekends. Before diagnosis, he used to take long walks with his dog, but now he feels too tired. Follow-up visits in the hospital do not show any signs of recurrence. He discusses these symptoms with a general practitioner (GP), who suggests referral to an oncological physiotherapist for rehabilitation. However, he does not want this because he worries about the intensity of the training, the travel time and the financial costs. He would prefer a programme in which he can choose the time, location, type and duration of physical activity.

GP care provision in the Netherlands is fully covered by health insurance, thereby increasing accessibility. However, little is known about how to implement such a programme in primary care, or indeed, the barriers and facilitators associated with implementation.

This study aims to implement and evaluate a homebased PA counselling programme in general practice for cancer survivors and to identify the barriers and facilitators related to the implementation.

\section{METHODS AND ANALYSES \\ Study design and setting}

This research, entitled the Stimulation of Daily Activity (SoDA) study, will have a single-arm longitudinal design to implement a PA counselling programme for cancer survivors in 15 general practices in the Netherlands from 2020 to 2024. Implementation research focuses on identifying the contextual factors that play a role in the implementation process, helping to clarify why and how an intervention works in a real world setting. ${ }^{14}{ }^{15} \mathrm{We}$ will constantly adapt the implementation strategy based on what works best for participating GPs, practice nurses (PNs) and patients, ${ }^{15}$ using the theoretical model of Grol and Wensing. ${ }^{16} 17$ This model relies on a concrete proposition for change and analysis of the current care situation, target population and setting. The process evaluation will use the RE-AIM framework (Reach, Effectiveness, Adoption, Implementation and Maintenance) ${ }^{18}$ which has been widely used to evaluate health behaviour interventions. ${ }^{19}$

\section{Study population}

Patients aged 18 years or older who finished their primary cancer treatment (ie, chemotherapy, surgery, radiotherapy or immunotherapy) at least 6 months ago will be eligible for inclusion, including those undergoing adjuvant hormonal therapy. We will exclude participants if they have had non-melanoma skin cancer, are participating in another PA programme (eg, in a hospital or rehabilitation centre), are terminally ill, or cannot take part (eg, physical or cognitive impairment, or any other reason, as judged by their GP). GPs and PNs will screen for eligible patients through their medical records or clinical visits. Eligible patients will receive an invitation from the GP's

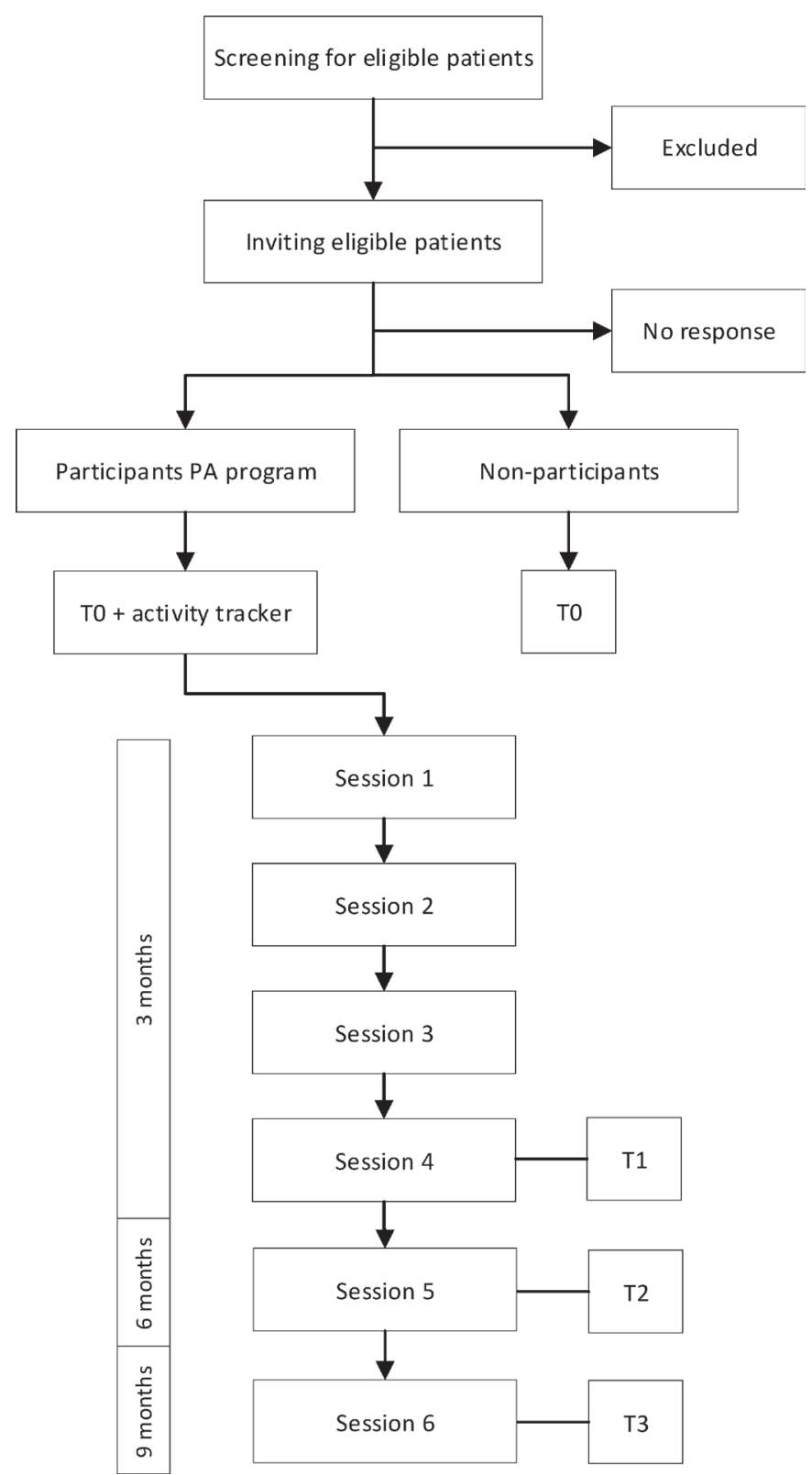

Figure 1 Study protocol flowchart. PA, physical activity.

practice by letter, telephone or in-person during routine GP or PN care. Patients who decide not to take part in the PA programme, but who consent to completing a questionnaire and analysis of their electronic patient records, will receive the baseline questionnaire (figure 1).

\section{Intervention}

The PA counselling programme is a home-based, personalised counselling protocol with a duration of 9 months that aims to motivate participants to increase PA in daily life (eg, walking, cycling or gardening). Entitled the COACH method, it uses strategies for behavioural change derived from the transtheoretical model,${ }^{20}$ the self-determination theory, ${ }^{21}$ the goal-setting theory, ${ }^{22}$ the implementation intention theory ${ }^{23}$ and the achievement goal theory. ${ }^{24}$ It has proven to be effective at increasing PA in patients with chronic obstructive pulmonary disease,,$^{25-27}$ in patients 
Table 1 Content of the coaching sessions in the practice nurse intervention

\begin{tabular}{|c|c|}
\hline Session & Content \\
\hline \multirow[t]{3}{*}{ S1 } & $\begin{array}{l}\text { Patient's motivation for PA is assessed using the SOC } \\
\text { questionnaire. }\end{array}$ \\
\hline & PA information from the activity tracker is synchronised. \\
\hline & SMART PA goals are set based on the baseline PA. \\
\hline \multirow[t]{5}{*}{ S2 } & PA information from the activity tracker is synchronised. \\
\hline & Achievement of the PA goal set during $S 1$ is discussed. \\
\hline & A new SMART PA goal is set. \\
\hline & Techniques for achieving the PA goal are discussed. \\
\hline & $\begin{array}{l}\text { A SMART PA goal for the peak performance day is set: } \\
\text { a self-selected day in which the patient aims to reach a } \\
\text { maximal PA target. }\end{array}$ \\
\hline \multirow[t]{4}{*}{ S3 } & PA information from the activity tracker is synchronised. \\
\hline & Achievement of the PA goal set during S2 is discussed. \\
\hline & The peak performance day is evaluated. \\
\hline & $\begin{array}{l}\text { A new SMART PA goal is set, which reflects participants' } \\
\text { personal activity norm. The personal activity norm lies } \\
\text { between the amount of PA on the peak performance day } \\
\text { and the regular amount of PA achieved at this current } \\
\text { session. }\end{array}$ \\
\hline \multirow[t]{3}{*}{ S4 } & PA information from the activity tracker is synchronised. \\
\hline & Achievement of the PA goal set during S3 is discussed. \\
\hline & $\begin{array}{l}\text { Principles of routinisation of behavioural change and } \\
\text { relapse prevention are discussed. }\end{array}$ \\
\hline \multirow[t]{4}{*}{ S5 } & PA information from the activity tracker is synchronised. \\
\hline & $\begin{array}{l}\text { The pattern of activity throughout the programme is } \\
\text { discussed. }\end{array}$ \\
\hline & $\begin{array}{l}\text { Adherence and self-management techniques to achieve } \\
\text { the personal activity norm in daily life are discussed. }\end{array}$ \\
\hline & $\begin{array}{l}\text { New strategies to enhance PA are introduced and } \\
\text { discussed. }\end{array}$ \\
\hline \multirow[t]{4}{*}{ S6 } & PA information from the activity tracker is synchronised. \\
\hline & The pattern of activity throughout the year is evaluated. \\
\hline & $\begin{array}{l}\text { The influence of the seasons on the year cycle of PA is } \\
\text { discussed. }\end{array}$ \\
\hline & $\begin{array}{l}\text { Techniques for maintaining PA and motivation for PA are } \\
\text { discussed. }\end{array}$ \\
\hline
\end{tabular}

PA, physical activity; SMART, specific, measurable, achievable, relevant and time bound; SOC, Stage of Change questionnaire.

undergoing haemodialysis, ${ }^{28}$ in women with obesity ${ }^{29}$ and in adult survivors of childhood cancer. ${ }^{30}$

In this study, the COACH method will comprise six coaching sessions of $30 \mathrm{~min}$ with the PN working at the patients' general practice (figure 1, table 1). Half of the sessions (S1, S4, S6) will take place at the general practice, the other half of the sessions can also be taken place digitally or by telephone. We will use the Fitbit Charge 3 (2018 Fitbit, Inc) activity tracker for goal setting and feedback, with patients instructed to wear it for at least 1 week before each coaching session. Participants will be instructed not to change their PA behaviour before the first session so that the PN can assess their baseline activity. The first four sessions will target developing strategies to increase daily PA, with patients asked to set specific, measurable, achievable, relevant and time bound PA goals with the PN. These goals will include the daily number of steps, the type of PA, and the target day and time for completing the goals. To be realistic, we will aim for a maximum $25 \%$ increase from current PA levels. The final two sessions will focus on maintaining the new PA behaviour, by discussing how to embed and maintain PA in daily routines and how to deal with barriers or setbacks (table 1).

\section{Implementation strategy}

We will recruit GP practices by newsletters, mailings and personal contact, organising presentations, as needed, to inform interested GPs about the procedures. Experienced instructors (MHGdG and $\mathrm{FH}$ ) will train $\mathrm{PNs}$ to implement the COACH method. The PNs will also receive education from oncology nurses about common physical and psychosocial problems among cancer survivors.

After recruiting GP practices, we will implement the programme iteratively to tailor the implementation strategy to the users involved (ie, patients, GPs and PNs). This means that, for each GP practice, we will continuously monitor the implementation, adapt the implementation strategy if necessary and use the lessons learnt for future strategies. To tailor the implementation strategy to the patient group, we have included a patient consultation group from the inception of the study protocol. These patients review all patient materials and procedures (ie, the invitation letter, the information brochure and newsletters) to enhance understanding. We will also individualise the PA programme to patients' needs regarding the activity types and goals. Patients will be free to use their own activity tracker, provided it measures both number of steps and calorie expenditure. For the PNs, they are free to adapt coaching sessions based on their own knowledge, experience or personal coaching style, provided they incorporate the core elements of the programme (see table 1). GPs and PNs can choose how to invite patients to the programme (eg, by letter, telephone or in-person).

We will assist GPs and PNs throughout the implementation, both individually and in groups, and will stimulate experience exchange. This will include regularly providing GPs, PNs and patients with feedback by newsletters designed to increase and maintain enthusiasm. GPs and PNs will receive practice-specific results and benchmarks of the number of participants they have included compared with other practices. GPs will receive a financial allowance to compensate for the work of the PN.

\section{Process evaluation of the implementation}

We will use the RE-AIM framework for the process evaluation, ${ }^{18}$ evaluating the five different interacting dimensions (RE-AIM) at the setting (GP practice) level and/or the individual (patient) level. Table 2 shows the measurement instruments and their timings for evaluating each RE-AIM dimension. Patient measurements at S1-S6 are 
Table 2 An overview of the measures and the measurement instruments used to evaluate the dimensions of the RE-AIM framework

\begin{tabular}{|c|c|c|c|}
\hline $\begin{array}{l}\text { RE-AIM } \\
\text { dimension }\end{array}$ & Measures & Measurement instrument & Time \\
\hline \multicolumn{4}{|l|}{ Reach } \\
\hline \multirow{15}{*}{ Individual } & Participating vs invited & & \\
\hline & Demographic information & Questionnaire & T0 \\
\hline & Level of education & & \\
\hline & Employment status & & \\
\hline & Living situation & & \\
\hline & Care received at home & & \\
\hline & Motivation for PA & $\mathrm{SOC}^{*}$ & $\mathrm{~S} 1 / \mathrm{TO}$ \\
\hline & Self-reported PA & IPAQ short form & TO \\
\hline & Clinical characteristics & GPs records & TO \\
\hline & Cancer diagnoses & & \\
\hline & Cancer stage & TNM staging & \\
\hline & Time since diagnosis & & \\
\hline & Type of treatment & & \\
\hline & Time since treatment & & \\
\hline & Comorbidities & $\mathrm{CCl}$ & \\
\hline
\end{tabular}

\section{Effectiveness}

Individual

$$
\text { Primary health outcomes }
$$

$$
\text { Fatigue }
$$

Fatigue

Depression

HADS-D

Anxiety

HADS-A

T0-T3

Secondary health outcomes

Number of steps

Calorie expenditure

Height

Weight

Lower limb strength

Aerobic endurance

Self-reported PA

Quality of life

Experiences PA programme
Activity tracker

S1-S6

Activity tracker

S1-S6

Measuring tape

S1

Scale

30 s sit-to-stand test

$\mathrm{S} 1, \mathrm{~S} 4, \mathrm{~S} 6$

$\mathrm{S} 1, \mathrm{~S} 4, \mathrm{~S} 6$

2 min step test

S1, S4, S6

IPAQ short form

T0, T1, T3

FACT-G

TO-T3

$\mathrm{T} 1-\mathrm{T} 3$

Questionnaire

Focus groups and individual interviews participants

Documentation

Questionnaire
Participating vs contacted GP practices

Practice characteristics

No. patients, GPs and PNs

Location of GP practice (urban/non-urban) 


\begin{tabular}{|c|c|c|c|}
\hline \multirow[t]{2}{*}{$\begin{array}{l}\text { RE-AIM } \\
\text { dimension }\end{array}$} & Measures & Measurement instrument & Time \\
\hline & Reasons for GP practices not participating & Documentation & TO \\
\hline \multicolumn{4}{|c|}{ Implementation } \\
\hline Individual & Accomplishment PA goals & Registration by PN & S2-S4 \\
\hline \multirow[t]{2}{*}{ Setting } & Adherence of the PN to the protocol & Observation checklist & $\mathrm{S} 1-\mathrm{S} 6$ \\
\hline & Quality of PN training for the $\mathrm{COACH}$ method & Questionnaire & Before T0 \\
\hline \multicolumn{4}{|c|}{ Maintenance } \\
\hline
\end{tabular}

${ }^{*}$ For participants, the practice nurse asked about the measure in $\mathrm{S} 1$; for non-participants, this was based on self-report at T0.

CCI, Charlson Comorbidity Index; FACT-F or -G, Functional Assessment of Cancer Therapy-Fatigue or General; GP, general practitioner; HADS-A or -D, Hospital Anxiety and Depression Scale-Anxiety or Depression; IPAQ, International Physical Activity Questionnaire; PA, physical activity; PN, practice nurse; RE-AIM, Reach, Effectiveness, Adoption, Implementation and Maintenance; SOC, Stage of Change questionnaire; TNM staging, TNM (tumour, node, metastases) Classification of Malignant Tumours.

assessed by the PN; patient measurements at T0-T3 are assessed by self-report questionnaires.

\section{Reach}

The participation rate and representativeness of participants will be used to evaluate reach. Participation rate will measure the number of patients who take part in the programme in relation to the number invited. Representativeness will measure demographic, lifestyle and clinical characteristics (see table 2). We will compare these characteristics between participants and non-participants, and we will evaluate the reasons given by both GPs for not inviting patients and the reasons for non-participants not taking part.

\section{Effectiveness}

The changes in health outcomes and participant experience of the PA programme will be used to evaluate effectiveness. The primary health outcomes are fatigue, anxiety and depression. We will use the Functional Assessment of Cancer Therapy-Fatigue (FACT-F) questionnaire, ${ }^{31}$ a valid and reliable tool for measuring fatigue in cancer survivors. ${ }^{31} 32$ It comprises 13 items that can give a total score of $0-52$, with lower scores suggesting greater fatigue. A three-point difference in the FACT-F will indicate a clinically important difference. ${ }^{33}$ We will use the Hospital Anxiety and Depression Scale, ${ }^{34}$ which has shown adequate psychometric properties for measuring symptoms of anxiety and depression in cancer survivors. ${ }^{35-37}$ It comprises seven items each in a depression and an anxiety subscale, with possible scores ranging from 0 to 21 per subscale and higher scores indicating greater symptoms. A difference of 1.50 in both subscales will indicate a clinically important difference. $^{38} 39$

Secondary health outcomes include average step count and calorie expenditure per day of the week before each coaching session (measured with the activity tracker), height, weight, lower limb strength, aerobic endurance, self-reported PA and quality of life. The $30 \mathrm{~s}$ sit-to-stand test and the 2 min step test will be assessed by the PN to measure lower limb strength and aerobic endurance, respectively. ${ }^{40}$ Both instruments have adequate validity in older adults. ${ }^{41}$ The short form of the International Physical Activity Questionnaire, which has shown sufficient reproducibility and construct validity, will be used to measure self-reported PA. ${ }^{42}$ Quality of life will be measured with the Functional Assessment of Cancer Therapy-General (FACT-G) questionnaire, ${ }^{32}$ which has also been shown to be reliable and valid for cancer patients. ${ }^{43}$ All measurements will be performed, as stated in table 2 , at baseline (T0), 3 months (T1), 6 months (T2) or 9 months (T3) or at session 1 (baseline), session 2 (3 weeks), session 3 (6 weeks), session 4 (3 months), session 5 (6 months) or session 6 (9 months).

We will evaluate participant experience of the PA programme through short questionnaires, focus groups and/or individual interviews. Literature review and expert opinion will be used to develop the topic list for the interviews and focus groups. Patient selection for the focus groups will use purposive sampling by general practice membership, motivation for PA (Stage of Change score $\left.^{44}\right)$, age, sex, educational level and change in PA from $\mathrm{T} 0$ to $\mathrm{T} 1$.

\section{Adoption}

The participation rate and representativeness of general practices, together with the barriers and facilitators experienced by GPs and PNs when implementing the programme, will inform our evaluation of adoption. We will calculate the participation rate as the number of practices taking part in relation to the number of practices contacted. We will assess representativeness based on the number of registered patients per practice, the number 
of GPs and PNs working at the practice, and whether the practice is urban/non-urban. Using focus groups and individual interviews, we will also evaluate the barriers and facilitators among GPs and PNs related to adopting the PA programme. Literature review and expert opinion will be used to develop the topic list for the interviews and focus groups. We will invite all GPs and PNs to the focus groups and will interview a selection by purposive sampling, considering practice size, GP or PN age and sex, and numbers of patients taking part. We will also evaluate the reasons why GP practices did not participate.

\section{Implementation}

We will evaluate implementation at the individual level by measuring participant adherence to attending the counselling sessions, wearing the activity tracker and accomplishing the PA goals. At the setting level, we will observe two sessions for each PN (one each from sessions 1-4 and 5-6) to assess the extent to which PNs adhere to the COACH protocol. Finally, we will evaluate whether the training adequately prepared the PNs to deliver the $\mathrm{COACH}$ method.

\section{Maintenance}

To evaluate maintenance, we will assess the use of the PA programme in GP practices 6 months after the study ends.

\section{Data-analyses}

\section{Quantitative analyses}

We will describe the RE-AIM data descriptively. Depending on the data distribution, we will use independent t-tests or non-parametric equivalents to measure differences between participants and non-participants. Linear mixed model analyses will be used to evaluate differences in outcomes over time. This approach allows missing data to be included in the analyses because it includes differences in the number of repeated measurements across individuals when values are missing at random. ${ }^{45}{ }^{46}$ We will include three levels in the model-general practice, patient and measurement time-and will use random intercepts at the general practice and patient levels to allow for differences between settings and individuals. Time will be a fixed effect dummy variable, with $\mathrm{T} 0$ set as a reference.

\section{Qualitative analyses}

The focus groups and individual interviews will be audio recorded, transcribed verbatim and anonymised. We will check audio recordings for inaccuracies and to enhance semantic understanding. Two researchers will perform thematic analyses by independently marking relevant transcript segments (inductive coding), identifying codes and themes, discussing any discrepancies until consensus and consulting a third researcher if necessary (open, axial and selective coding). Using an iterative process, we will include new information in the topic lists for future interviews. If no more subthemes emerge from five interviews, we will assume data saturation and will not organise further interviews or focus groups.

\section{Patient and public involvement}

The study protocol was developed with extensive engagement of a patient consultation group from the Dutch Breast Cancer Patient Organisation (Borstkanker Vereniging Nederland). This patient consultation group highlighted that PNs should have adequate understanding about the physical and psychosocial problems that cancer survivors encounter. We therefore complemented the training of PNs with education from oncology nurses. Two patient partners from this patient consultation group are also incorporated in our research project team. They will review all patient materials and procedures (ie, the invitation letter, the information brochure, and newsletters) to enhance understanding. They also actively participate in the project meetings where we discuss the progress, the study results and the dissemination of the study results.

\section{Ethics and dissemination}

The Medical Research Ethics Committee of the University Medical Centre Groningen, the Netherlands, concluded that this study was not subject to the Dutch Medical Research Involving Human Subjects Act (registration number: 201900586). All recruited participants will be required to give written informed consent after we fully explain the procedures. We will make the study results available to patients and GPs and other interested medical health professionals or health policy makers via international and national peer-reviewed journals, international and national conferences, newsletters, public summaries, and (social) media.

\section{DISCUSSION}

The SoDA study will evaluate the implementation of a PA counselling programme among cancer survivors in Dutch primary care. We hope the programme will increase daily PA in this cohort, especially among those with sedentary or unhealthy lifestyles, and reduce symptoms of fatigue, depression and anxiety. Contrasting with experimental research, which aims to assess the effects of an intervention in a controlled setting, implementation research focuses on identifying contextual factors that play a role in why and how an intervention works in a real world setting. ${ }^{145}$ GPs, PNs and patients taking part in this research will influence the implementation strategy as we continuously adapt it to their needs. ${ }^{15}$

In this study, we chose a single-arm longitudinal study design rather than a pragmatic or cluster randomised trial. ${ }^{15}$ We consider that systematic reviews and metaanalysis have adequately confirmed the favourable impact of PA interventions on various health outcomes in cancer survivors (eg, fatigue, depression, anxiety and physical function).${ }^{1-8}$ Concerning the PA counselling programme in this study, recent meta-analyses have shown that comparable home-based PA interventions favourably affect physical function and fatigue in cancer survivors. ${ }^{18}$ Although a controlled design would generate more certainty about the effect of the intervention on patient health outcomes, 
which is an important limitation of this study, our participatory design and process evaluation will provide more information on the factors contributing to the success of implementation.

Implementing a new intervention in primary care can be complex and comes with many challenges, especially during the COVID-19 pandemic. We expect to encounter barriers during implementation, such as GPs and PNs having insufficient time due to their usual clinical work. GPs and PNs may therefore be less willing to take part, and if they do, may have little time to invite patients or schedule counselling sessions. Patients may also be less willing to take part due to national travel limitations or fear of getting COVID-19. These barriers might limit patient recruitment and, ultimately, the implementation process. It is essential that we not only offer extensive support to GPs and PNs but also monitor the implementation strategy so that we can adapt to problems early.

The SoDA study will provide new insights into the factors that play a role in implementing a PA programme in general practice. This will guide the implementation of lifestyle programmes in primary care in the future.

Acknowledgements We would like to thank Michiel de Boer for help with setting up the statistical analysis plan. We also thank Dr Robert Sykes (www.doctored. org.uk) for providing technical editing and writing services for the final draft of this manuscript.

Contributors Conceptualisation: $\mathrm{AB}, \mathrm{MHGdG}, \mathrm{GHdB}, \mathrm{MYB}$ and DB. Methodology: FH, N-DLW, AB, AW, MHGdG, GHdB, MYB and DB. Data curation: FH and DB. Writing - original draft: $F H$ and DB. Writing - review and editing: N-DLW, AB, AW, MHGdG, GHdB, MYB and DB. Visualisation: FH. Supervision: N-DLW, MYB and DB. Project administration: FH, MYB and DB. Funding acquisition: $A B, M H G d G$ and DB. All authors have read and agreed to the published version of the manuscript.

Funding The Dutch Cancer Society (KWF Kankerbestrijding; grant number 12375, 2019-1) will support this work.

\section{Competing interests None declared.}

Patient and public involvement Patients and/or the public were involved in the design, or conduct, or reporting, or dissemination plans of this research. Refer to the Methods section for further details.

Patient consent for publication Not applicable.

Provenance and peer review Not commissioned; peer reviewed for ethical and funding approval prior to submission.

Open access This is an open access article distributed in accordance with the Creative Commons Attribution Non Commercial (CC BY-NC 4.0) license, which permits others to distribute, remix, adapt, build upon this work non-commercially, and license their derivative works on different terms, provided the original work is properly cited, appropriate credit is given, any changes made indicated, and the use is non-commercial. See: http://creativecommons.org/licenses/by-nc/4.0/.

ORCID iD

Famke Huizinga http://orcid.org/0000-0001-9560-2394

\section{REFERENCES}

1 Swartz MC, Lewis ZH, Lyons EJ, et al. Effect of Home- and community-based physical activity interventions on physical function among cancer survivors: a systematic review and meta-analysis. Arch Phys Med Rehabil 2017;98:1652-65.

2 Mishra SI, Scherer RW, Geigle PM. Exercise interventions on healthrelaated quality of life for cancer survivors. Cochrane Database Syst Rev 2012.

3 Cramp F, Daniel J. Exercise for the management of cancer-related fatigue in adults. Cochrane Database Syst Rev 2008:CD006145.
4 McMillan EM, Newhouse IJ. Exercise is an effective treatment modality for reducing cancer-related fatigue and improving physical capacity in cancer patients and survivors: a meta-analysis. Appl Physiol Nutr Metab 2011;36:892-903.

5 Craft LL, Vaniterson EH, Helenowski IB, et al. Exercise effects on depressive symptoms in cancer survivors: a systematic review and meta-analysis. Cancer Epidemiol Biomarkers Prev 2012;21:3-19.

6 Brown JC, Huedo-Medina TB, Pescatello LS, et al. The efficacy of exercise in reducing depressive symptoms among cancer survivors: a meta-analysis. PLoS One 2012;7:e30955.

7 Herring MP, O'Connor PJ, Dishman RK. The effect of exercise training on anxiety symptoms among patients: a systematic review. Arch Intern Med 2010;170:321-31.

8 Huizinga F, Westerink N-DL, Berendsen AJ, et al. Home-Based physical activity to alleviate fatigue in cancer survivors: a systematic review and meta-analysis. Med Sci Sports Exerc 2021;53:2661-74.

9 Coletta AM, Marquez G, Thomas P, et al. Clinical factors associated with adherence to aerobic and resistance physical activity guidelines among cancer prevention patients and survivors. PLOS One 2019;14:e0220814.

10 Kampshoff CS, van Mechelen W, Schep G, et al. Participation in and adherence to physical exercise after completion of primary cancer treatment. Int J Behav Nutr Phys Act 2016;13:100.

11 Ormel HL, van der Schoot GGF, Sluiter WJ, et al. Predictors of adherence to exercise interventions during and after cancer treatment: a systematic review. Psychooncology 2018;27:713-24.

12 Hardcastle SJ, Maxwell-Smith C, Kamarova S, et al. Factors influencing non-participation in an exercise program and attitudes towards physical activity amongst cancer survivors. Support Care Cancer 2018;26:1289-95.

13 Etnier JL, Karper WB, Park S-Y, et al. Motivating mature adults to be physically active. J Aging Phys Act 2017;25:325-31.

14 Hwang S, Birken SA, Melvin CL, et al. Designs and methods for implementation research: advancing the mission of the CTSA program. J Clin Trans/ Sci 2020;4:159-67.

15 Peters $\mathrm{DH}$, Adam T, Alonge O. Implementation research: what it is and how to do it. BMJ 2013;347:731-6.

16 Grol R, Wensing M. Implementatie: effectieve verbeteringen van patientenzorg. 6th ed. Bohn Stafleu van Loghum, 2016.

17 Grol R, Grimshaw J. From best evidence to best practice: effective implementation of change in patients' care. Lancet 2003;362:1225-30

18 Glasgow RE, Vogt TM, Boles SM. Evaluating the public health impact of health promotion interventions: the RE-AIM framework. Am J Public Health 1999;89:1322-7.

19 Gaglio B, Shoup JA, Glasgow RE. The RE-AIM framework: a systematic review of use over time. Am J Public Health 2013;103:e38-46.

20 Prochaska JO, DiClemente CC. Transtheoretical therapy: toward a more integrative model of change. Psychotherapy: Theory, Research \& Practice 1982;19:276-88.

21 Deci EL, Ryan RM. The "What" and "Why" of Goal Pursuits: Human Needs and the Self-Determination of Behavior. Psychol Inq 2000;11:227-68.

22 Locke EA, Latham GP. Building a practically useful theory of goal setting and task motivation. A 35-year odyssey. Am Psychol 2002;57:705-17.

23 Gollwitzer PM, Sheeran P. Implementation intentions and goal achievement: a meta-analysis of effects and processes. Adv Exp Soc Psychol 2006;38:69-119.

24 Urdan T, Kaplan A. The origins, evolution, and future directions of achievement goal theory. Contemp Educ Psychol 2020;61:101862.

25 de Blok BMJ, de Greef MHG, ten Hacken NHT, et al. The effects of a lifestyle physical activity counseling program with feedback of a pedometer during pulmonary rehabilitation in patients with COPD: a pilot study. Patient Educ Couns 2006;61:48-55.

26 Hospes G, Bossenbroek L, Ten Hacken NHT, et al. Enhancement of daily physical activity increases physical fitness of outclinic COPD patients: results of an exercise counseling program. Patient Educ Couns 2009;75:274-8.

27 Altenburg WA, ten Hacken NHT, Bossenbroek L, et al. Short- and long-term effects of a physical activity counselling programme in COPD: a randomized controlled trial. Respir Med 2015;109:112-21.

28 van Vilsteren MCBA, de Greef MHG, Huisman RM. The effects of a low-to-moderate intensity pre-conditioning exercise programme linked with exercise counselling for sedentary haemodialysis patients in the Netherlands: results of a randomized clinical trial. Nephrol Dial Transplant 2005;20:141-6.

29 Mutsaerts MAQ, van Oers AM, Groen $\mathrm{H}$, et al. Randomized trial of a lifestyle program in obese infertile women. $N$ Engl $J$ Med 2016;374:1942-53. 
30 Blaauwbroek R, Bouma MJ, Tuinier W, et al. The effect of exercise counselling with feedback from a pedometer on fatigue in adult survivors of childhood cancer: a pilot study. Support Care Cancer 2009;17:1041-8

31 Yellen SB, Cella DF, Webster K, et al. Measuring fatigue and other anemia-related symptoms with the functional assessment of cancer therapy (fact) measurement system. J Pain Symptom Manage 1997;13:63-74.

32 Cella DF, Tulsky DS, Gray G, et al. The functional assessment of cancer therapy scale: development and validation of the general measure. J Clin Oncol 1993;11:570-9.

33 Cella D, Eton DT, Lai J-S, et al. Combining anchor and distributionbased methods to derive minimal clinically important differences on the functional assessment of cancer therapy (fact) anemia and fatigue scales. J Pain Symptom Manage 2002;24:547-61.

34 Zigmond AS, Snaith RP. The hospital anxiety and depression scale. Acta Psychiatr Scand 1983;67:361-70.

35 Katz MR, Kopek N, Waldron J, et al. Screening for depression in head and neck cancer. Psychooncology 2004;13:269-80.

36 Cameron IM, Crawford JR, Lawton K, et al. Psychometric comparison of PHQ-9 and HADS for measuring depression severity in primary care. Br J Gen Pract 2008;58:32-6.

37 Vodermaier A, Linden W, Siu C. Screening for emotional distress in cancer patients: a systematic review of assessment instruments. $J$ Natl Cancer Inst 2009;101:1464-88.

38 Puhan MA, Frey M, Büchi S, et al. The minimal important difference of the hospital anxiety and depression scale in patients with chronic obstructive pulmonary disease. Health Qual Life Outcomes 2008;6:46-6.

39 Smid DE, Franssen FME, Houben-Wilke S, et al. Responsiveness and MCID estimates for cat, CCQ, and HADS in patients with COPD undergoing pulmonary rehabilitation: a prospective analysis. $J \mathrm{Am}$ Med Dir Assoc 2017:18:53-8.

40 Jones CJ, Rikli RE. Measuring functional fitness in older adults. J Act Ageing 2002:24-30.

41 Rikli RE, Jones CJ. Development and validation of a functional fitness test for Community-Residing older adults. J Aging Phys Act 1999;7:129-61.

42 Poppel MNMvan, Paw CA. Reproduceerbaarheid en validiteit van de Nederlandse versie van de international physical activity questionnaire (IPAQ). TSG 2004;82:457-62.

43 Overcash J, Extermann M, Parr J, et al. Validity and reliability of the FACT-G scale for use in the older person with cancer. Am J Clin Oncol 2001;24:591-6.

44 Prochaska JO, DiClemente CC. Stages of change in the modification of problem behaviors. Prog Behav Modif 1992;28:183-218.

45 Magezi DA. Linear mixed-effects models for within-participant psychology experiments: an introductory tutorial and free, graphical user interface (LMMgui). Front Psychol 2015;6:2.

46 Baayen RH, Davidson DJ, Bates DM. Mixed-Effects modeling with crossed random effects for subjects and items. J Mem Lang 2008;59:390-412. 